\title{
Welchen Einfluss hat die Aufteilung von Hausarbeit und Kinderbetreuung auf Partnerschaftskonflikte und Partnerschaftszufriedenheit bei berufstätigen Paaren? ${ }^{1}$
}

\section{Division of housework and child care, conflict, and relationship satisfaction in dual-earner couples}

\begin{abstract}
Zusammenfassung:
In bisherigen Studien zeigte sich, dass vor allem bei Frauen die Zufriedenheit mit der Arbeitsteilung mit der Partnerschaftszufriedenheit zusammenhängt. Ungeklärt ist, ob dieser Zusammenhang durch eine Reduktion des Konfliktpotentials in der Partnerschaft vermittelt wird und welche Rolle dabei die tatsächliche Aufteilung der Familienarbeit spielt. 207 Schweizer Doppelverdiener-Paare mit Kleinkindern wurden anhand von Fragebogen zu Partnerschaft und Arbeitsteilung befragt. Die familiäre Arbeitsteilung wurde überwiegend als zufriedenstellend erlebt, dabei verrichteten mehrheitlich die Frauen die Hausarbeit und betreuten die Kinder. Bei beiden Partnern war eine geringere $\mathrm{Zu}$ friedenheit mit der Aufteilung von Hausarbeit und Kinderbetreuung mit häufigeren Konflikten assoziiert, welche wiederum mit einer geringeren Partnerschaftszufriedenheit zusammenhingen. Im Gegensatz zur Kinderbetreuung waren die Effekte der Arbeitsteilung der Hausarbeit auf die Partnerschaft stärker für die Frauen als für die Männer. Dabei war die subjektive Bewertung der Arbeitsteilung bedeutender für die berichteten Konflikte und die Partnerschaftszufriedenheit als die tatsächliche Arbeitsteilung.
\end{abstract}

\begin{abstract}
:
Although women's relationship satisfaction is known to covary with satisfaction regarding couples' division of housework and child care, the factors mediating these associations are rarely examined. To test the hypothesis that relationship conflict mediates the link between housework and relationship satisfaction, 207 dual-earner couples with young children completed questionnaires about their relationship and how they divided housework and child care. Most couples were satisfied with the division of labor, and women did most of the housework and child care. For husbands and wives, dissatisfaction with division of housework and child care was associated with more perceived conflicts, which in turn covaried with lower relationship satisfaction. Division of housework was more strongly associated with relationship satisfaction for women than for men, but there were no gender differences for the impact of child care responsibilities on relationship satisfaction. In general, subjective evaluation of the division of labor had stronger effects on relationship conflicts and satisfaction than the division of labor itself.
\end{abstract}

1 Die vorliegende Untersuchung wurde im Rahmen des von der Europäischen Union geförderten Verbundprojekts FamWork durchgeführt, in dem die Vereinbarkeit von Familie und Beruf in verschiedenen europäischen Ländern untersucht wurde (Projekt HPSE-CT-2002-00147. http://www.eu-projectfamwork.org). Die Schweizerische Projektgruppe (M. Perrez/P. Wilhelm) wurde vom Schweizerischen Nationalfonds gefördert (Projekt Nr. 109324), bei dem wir uns an dieser Stelle herzlich bedanken. Bedanken möchten wir uns auch bei Dr. Dominik Schöbi, der die Datenerhebung organisiert hat. 
Schlagwörter: Arbeitsteilung, Hausarbeit, Kinderbetreuung, Partnerschaftszufriedenheit, Doppelverdiener
Key words: division of labor, housework, child care, relationship satisfaction, dual-earners

Die Erwerbsquote von Frauen ist in den letzen Jahrzehnten kontinuierlich angestiegen (Hofäcker 2007). Der größere Anteil, den Frauen heute an der Erwerbsarbeit leisten, hat jedoch nicht zu einer wesentlichen Veränderung der Aufteilung der familiären Arbeit geführt. Amerikanische und europäische Studien belegen übereinstimmend, dass erwerbstätige Frauen zwei bis dreimal so viel Hausarbeit verrichten, wie ihre Partner (Bartley et al. 2005; Coltrane 2000; Hoelter 2002; Hofäcker 2007; Kirchler/Venus 2000). Besonders die täglich anfallenden Tätigkeiten, wie Kochen, Putzen oder Kleiderwaschen gehen zu Lasten der Frauen (Coltrane 2000; Hofäcker 2007; Koppetsch/Burkart 1999). Auch für die Betreuung der Kinder sind hauptsächlich die Mütter zuständig und übernehmen in der Regel 60 bis $80 \%$ des erforderlichen Aufwands (Ehrenberg et al. 2001; Grote et al. 2002; Hofäcker 2007).

Die familiäre Arbeitsteilung zu Ungunsten der Frauen wird abhängig vom theoretischen Bezugsrahmen auf unterschiedliche Faktoren zurückgeführt. Ökonomisch ausgerichtete Theorien stützen sich dabei primär auf Kosten-Nutzen-Überlegungen, wonach die Arbeitsteilung sich aus den unterschiedlichen Ressourcen (bspw. Ausbildung, Einkommen) beider Partner ergibt. Allerdings zeigte sich in verschiedenen Studien, dass die Ressourcen der Partner die Arbeitsteilung nur zu einem geringen Anteil erklären (u.a. Levy/ Ernst 2002; Schulz/Blossfeld 2006) und der Einfluss verschiedener Ressourcen geschlechtsabhängig war (Huinink/Reichart 2008; Klaus/Steinbach 2002; Lothaller et al. 2009; Rohmann et al. 2002). Soziologische Theorien hingegen, welche gesellschaftliche Bedingungen und Normen in den Vordergrund stellen, erklären die Asymmetrie der Geschlechter in Bezug auf die Aufteilung der Familienarbeit besser (Coltrane 2000; Schulz/Blossfeld 2006).

Die Diskussion darüber, wie die Ungleichverteilung der familiären Arbeit erklärt werden kann, soll hier nicht weiter vertieft werden, da die vorliegende Arbeit nicht auf Ursachen fokussiert (einen Überblick über die verschiedenen theoretischen Ansätze geben z.B. Grunow 2007; Huinink/Reichart 2008; Schulz/Blossfeld 2006). Im Fokus unserer psychologischen Studie stehen vielmehr die Folgen, die sich aus der ungleichen Aufteilung der familiären Arbeit für die Qualität der Paarbeziehung ergeben. Untersucht werden soll, wie sich a) die Aufteilung der Familienarbeit zwischen den Partnern sowie b) die subjektive Bewertung dieser Aufteilung auf Konflikte in der Partnerschaft und die Partnerschaftszufriedenheit auswirken.

\section{Zufriedenheit mit der Aufteilung der familiären Arbeit}

In verschiedenen Studien zeigte sich, dass trotz der ungleichen Lastenverteilung sowohl Männer als auch Frauen mit der in ihrer Partnerschaft bestehenden Arbeitsteilung mehrheitlich zufrieden waren (u.a. Chan et al. 1998; Kluwer et al. 1996). Allerdings war die Zufriedenheit der Männer, die überwiegend auch die Nutznießer der Ungleichverteilung sind, höher als die der Frauen (Kirchler/Venus 2000; Stevens et al. 2001, 2005). 
Während für Männer die Zufriedenheit mit der Arbeitsteilung nicht davon abhing, wie viel Hausarbeit sie selbst verrichteten, war die Zufriedenheit der Frauen umso höher, je mehr sich ihre Partner für die Hausarbeit engagierten (Stevens et al. 2001). Dabei waren Beiträge der Männer zur Bewältigung der ,täglichen Arbeitslast“ (z.B. Geschirr abwaschen oder Putzen) für die Zufriedenheit der Frauen wichtiger als sporadische Tätigkeiten (z.B. Reparaturarbeiten oder Einkaufsgänge) oder ihr eigener Anteil an der Hausarbeit (Himsel/Goldberg 2003; Hochschild 1989).

\section{Familiäre Arbeitsteilung, Partnerschaftskonflikte und Partnerschaftszufriedenheit}

Wenn Frauen unzufrieden mit der Arbeitsteilung waren, gab es mehr Konflikte in der Partnerschaft. Für Männer wurde dieser Zusammenhang nicht beobachtet (Kluwer et al. 1996). Ein weiteres Ergebnis der Studie von Kluwer und Mitarbeitern war, dass Paare mit Kindern eine geringere Zufriedenheit mit der Arbeitsteilung und mehr Konflikte berichteten als kinderlose Paare. Die Partnerschaftskonflikte drehten sich dabei häufiger um die Aufteilung der Hausarbeit als um die Aufteilung der Erwerbsarbeit.

Der Anteil, den der Partner bei der Erledigung der täglichen Hausarbeit übernimmt, und die damit zusammenhängende Zufriedenheit mit der Aufteilung der Hausarbeit, ist insbesondere bei Frauen mit der Partnerschaftszufriedenheit assoziiert (Erickson 1993; Essex/Hong 2005; Stevens et al. 2001). In der Untersuchung von Stevens und Mitarbeitern (2001) hing die Zufriedenheit mit der Aufteilung der Hausarbeit bei beiden Partnern positiv mit der Partnerschaftszufriedenheit zusammen. Zudem waren Frauen mit ihrer Partnerschaft umso zufriedener, je weniger Hausarbeit sie selber verrichteten. Bezogen auf die Kinderbetreuung zeigte sich, dass ein verstärktes Engagement der Väter mit einer höheren Partnerschaftszufriedenheit beider Partner assoziiert war (Bonney et al. 1999; Erickson 1993).

In Untersuchungen zur Gerechtigkeitswahrnehmung ergaben sich ähnliche Ergebnisse. Je ungerechter die Arbeitsteilung erlebt wurde, umso geringer war die Partnerschaftszufriedenheit und längerfristig auch die Stabilität der Beziehung (Blair 1998; Frisco/Williams 2003). Die empfundene Gerechtigkeit bezogen auf die Verteilung der Hausarbeit hing dabei stärker mit der Partnerschaftszufriedenheit zusammen als die tatsächliche Arbeitsteilung (u.a. Blair 1998; Mikula et al. 2008; Voydanoff/Donnelly 1999).

\section{Die vorliegende Studie}

Besonders in Familien mit Kleinkindern stellen die Mehrfachbelastungen, die sich aus den Erfordernissen der beruflichen und familiären Arbeit ergeben, ein großes Konfliktpotential dar. Deshalb wurden in der vorliegenden Studie Paare mit Kleinkindern untersucht, bei denen beide Partner erwerbstätig waren. Im Unterschied zu den meisten Studien, die sich bisher mit dem Thema beschäftigt haben, wurden sämtliche Informationen und Einschätzungen von beiden Partnern erhoben und in ein Modell integriert, das es erlaubt, intrapersonelle von interpersonellen Effekten zu trennen. Dabei werden die 
Aufteilung der Hausarbeit und die Aufteilung der Kinderbetreuung getrennt voneinander betrachtet, wie dies von Coltrane und Kollegen seit längerem gefordert wird (Coltrane 2000; Ishii-Kuntz/ Coltrane 1992). Ziel ist es, zu untersuchen, wie die Aufteilung der Hausarbeit bzw. der Kinderbetreuung die Partnerschaftszufriedenheit von Frauen und Männern beeinflusst, und ob dieser Zusammenhang durch die subjektive Bewertung der Arbeitsteilung und die wahrgenommene Konflikthäufigkeit vermittelt wird.

In Abbildung 1 ist dargestellt, wie die Aufteilung der Erwerbs- und Familienarbeit, sowie die Zufriedenheit mit dieser Aufteilung, mit der Wahrnehmung von Partnerschaftskonflikten und der Partnerschaftszufriedenheit gemäß unserer Erwartung zusammenhängen. Bei unserem Modell handelt es sich um ein erweitertes Aktor-Partner-Interdependenz-Modell (APIM; Kenny et al. 2006), in dem die Angaben beider Partner gleichzeitig analysiert werden. Das dargestellte Modell impliziert folgende Annahmen: Die Aufteilung der Erwerbsarbeit beeinflusst die Aufteilung der Familienarbeit (d.h. der Hausarbeit bzw. der Kinderbetreuung) (Pfad $a$ ). Sowohl die Aufteilung der Erwerbsarbeit wie auch die Aufteilung der Familienarbeit haben einen Einfluss darauf, wie zufrieden Frauen (Pfad $b_{f}$ und $c_{f}$ ) bzw. Männer (Pfad $b_{m}$ und $c_{m}$ ) mit der Arbeitsteilung sind. Die eigene $\mathrm{Zu}$ friedenheit mit der Arbeitsteilung sagt wiederum vorher, wie konfliktreich Männer bzw. Frauen ihre Partnerschaft wahrnehmen (Aktoreffekt ${ }^{2} f_{f}$ bzw. $f_{m}$ ). Wie zufrieden die Frauen mit der Arbeitsteilung sind, kann jedoch auch einen Einfluss darauf haben, wie konfliktreich die Männer die Partnerschaft wahrnehmen (Partnereffekt $h_{m f}$ ). Ein solcher Partnereffekt ist auch in der umgekehrten Richtung möglich $\left(h_{f m}\right)$, d.h. die Zufriedenheit mit der Arbeitsteilung der Männer könnte beeinflussen, wie konfliktreich die Frauen ihre Partnerschaft erleben. Die berichteten Partnerschaftskonflikte sind sowohl mit der eigenen Partnerschaftszufriedenheit (Aktoreffekte $i_{f}$ und $i_{m}$ ) als auch mit der Partnerschaftszufriedenheit des Partners assoziiert (Partnereffekt $j_{m f}$ bzw. $j_{f m}$ ). Darüber hinaus ist ein direkter Effekt der Zufriedenheit mit der Aufteilung der Familienarbeit auf die Partnerschaftszufriedenheit möglich (Aktoreffekt $g_{f}$ bzw. $g_{m}$ ). Das Modell sieht ferner vor, dass die tatsächliche Aufteilung der Familienarbeit einen direkten Effekt auf die Konfliktwahrnehmung $\left(d_{f}\right.$ bzw. $\left.d_{m}\right)$ und die Partnerschaftszufriedenheit $\left(e_{f}\right.$ bzw. $\left.e_{m}\right)$ beider Partner haben kann.

In den nachfolgend aufgelisteten Hypothesen präzisieren wir die Beziehungen, die zwischen den Variablen im Modell bestehen, weiter:

H-1.1: Der Zusammenhang zwischen der Zufriedenheit mit der Arbeitsteilung und den selbstberichteten Partnerschaftskonflikten ist negativ und bei Frauen stärker ausgeprägt als bei Männern (Aktoreffekt: $f_{f}<f_{m}<0$ ).

H-1.2: Die Zufriedenheit mit der Arbeitsteilung ist a) bei Frauen und b) bei Männern negativ mit den vom Partner berichteten Konflikten assoziiert (Partnereffekt H-1.2a: $h_{m f}<$ $\left.0 ; \mathrm{H}-1.2 \mathrm{~b}: h_{f m}<0\right)$.

H-1.3: Der direkte Effekt der tatsächlichen Arbeitsteilung auf die Partnerschaftskonflikte ist a) bei Frauen und b) bei Männern kleiner als der indirekte Effekt, welcher über die $\mathrm{Zu}$ -

2 Als Aktoreffekte werden Zusammenhänge bezeichnet, die zwischen Variablen der gleichen Person bestehen, während Zusammenhänge, die zwischen Variablen unterschiedlicher Personen bestehen als Partnereffekte bezeichnet werden. Es handelt sich dabei um Netto-Effekte, d.h. aus dem Aktoreffekt ist der Einfluss des Partners herauspartialisiert und aus dem Partnereffekt der Einfluss des Aktors. 
friedenheit mit der Arbeitsteilung vermittelt wird (H-1.3a: $d_{f}<c_{f}^{*} f_{f}+\mathrm{c}_{m} * \mathrm{~h}_{f m} ; \mathrm{H}-1.3 \mathrm{~b}: d_{m}$ $\left.<c_{m}{ }^{*} f_{m}+\mathrm{c}_{f}{ }^{*} \mathrm{~h}_{m f}\right)$.

H-2.1: Die berichteten Partnerschaftskonflikte hängen a) bei Frauen und b) bei Männern negativ mit der eigenen Partnerschaftszufriedenheit zusammen (Aktoreffekt H-2.1a: $i_{f}<$ 0 ; H-2.1b: $i_{m}<0$ ).

H-2.2: Die berichteten Partnerschaftskonflikte hängen a) bei Frauen und b) bei Männern negativ mit Partnerschaftszufriedenheit des Partners zusammen (Partnereffekt H-2.2a: $j_{m f}$ $<0$; H-2.2b: $\left.j_{f m}<0\right)$.

H-3: Der Zusammenhang zwischen der Zufriedenheit mit der Arbeitsteilung und der Partnerschaftszufriedenheit wird a) bei Frauen und b) bei Männern über die berichteten Partnerschaftskonflikte vermittelt (H-3a: $\left.f_{f} * i_{f}>0 ; \mathrm{H}-3 \mathrm{~b}: f_{m} * i_{m}>0\right)$.

H-4: Die Partnerschaftszufriedenheit hängt a) bei Frauen und b) bei Männern stärker mit der Zufriedenheit mit der familiären Arbeitsteilung zusammen als mit der tatsächlichen Aufteilung der familiären Arbeit. D.h. die Summe der Effekte der tatsächlichen Arbeitsteilung auf die Partnerschaftszufriedenheit, welche über die Zufriedenheit mit der Arbeitsteilung vermittelt werden, ist größer als die Summe der Effekte, die nicht über die Zufriedenheit mit der Arbeitsteilung vermittelt sind (H-4a: $c_{f}{ }^{*} g_{f}+c_{f}^{*} f_{f}^{*} i_{f}+c_{f}{ }^{*} h_{m f}{ }^{*} j_{f m}+$ $c_{m}{ }^{*} f_{m}{ }^{*} j_{f m}+c_{m} * h_{f m}{ }^{*} i_{f}>e_{f}+d_{f} * i_{f}+d_{m}{ }^{*} j_{f m} ; \mathrm{H}-4 \mathrm{~b}: c_{m}{ }^{*} g_{m}+c_{m} * f_{m} * i_{m}+c_{m} * h_{f m}{ }^{*} j_{m f}+c_{f} * f_{f}^{*} j_{m f}$ $\left.+c_{f}^{*} h_{m f} * i_{m}>e_{m}+d_{m} * i_{m}+d_{f}^{*} j_{m f}\right)$.

\section{Methode}

\section{Stichprobe}

An der Studie nahmen 207 deutschsprachige Schweizer Paare teil, welche hauptsächlich über Anzeigen in Kinderkrippen und Zeitungsinserate in den Kantonen Bern und Fribourg/Freiburg sowie im Bekanntenkreis der Studienmitarbeiter rekrutiert wurden und die folgende Einschlusskriterien erfüllten: beide Partner a) wohnten in einem gemeinsamen Haushalt, b) waren mindestens 15 Stunden pro Woche erwerbstätig, c) hatten ein gemeinsames Kind zwischen ein und fünf Jahren, und d) waren beide bereit teilzunehmen. Den Paaren wurde der Fragebogen zugeschickt oder durch Studienmitarbeiter überreicht. Die Teilnehmer wurden gebeten, die Fragebogen unabhängig vom Partner auszufüllen und per Post an das Psychologische Departement zurück zu schicken. Für die Teilnahme erhielten die Paare 50 Schweizer Franken (ca. 30 Euro).

Die teilnehmenden Frauen waren im Mittel 35.5 Jahre $(S D=4.3)$ alt, die Männer 38 Jahre $(S D=5.3)$; $55 \%$ der Frauen und $64 \%$ der Männer waren Akademiker. Die Partner waren durchschnittlich seit 11.1 Jahren $(S D=4.7)$ in einer stabilen Partnerschaft und wohnten im Mittel seit 8.7 Jahren in einem gemeinsamen Haushalt $(S D=3.7) .86 .5 \%$ waren verheiratet und hatten zumeist zwei Kinder (im Durchschnitt hatten die Frauen 1.7 Kinder $(S D=.65)$, die Männer $1.8(S D=.76))$. Das mittlere Alter des jüngsten (gemeinsamen) Kindes betrug 2.4 Jahre $(S D=1.3)$. 


\section{Erfasste Konstrukte}

Schätzung des zeitlichen Aufwands für Berufs- und Familienarbeit. Die Teilnehmer schätzten ein, wie viele Stunden pro Woche sie selbst und wie viele Stunden ihr Partner für den Beruf (einschließlich des Arbeitswegs), für den Haushalt (Putzen, Kochen, Geschirrspülen, Wäschewaschen, Bügeln, Einkaufen usw.), für Instandhaltungs- und Reparaturarbeiten (z.B. Erledigen kleinerer Reparaturen, Gartenarbeit) und für die Kinderbetreuung (Wickeln, Baden, Essen geben, Spielen, bei Hausaufgaben helfen, Bring- und Abholdienste usw.) aufwenden. Für die drei Typen familiärer Arbeit (Haushalt, Instandhaltung und Kinderbetreuung) wurde zusätzlich erfasst, wie viele Stunden andere Personen (z.B. Haushaltshilfe oder andere Familienmitglieder) dafür aufwenden. Für jeden Tätigkeitsbereich wurden die Stundenzahl der Selbsteinschätzung sowie die vom Partner vorgenommene Fremdeinschätzung gemittelt ${ }^{3}$ (vgl. Wilkie et al. 1998). Da die Wochenstundenzahl für Instandhaltungs- und Reparaturarbeiten relativ gering war (Frauen: $M=$ 2.0, Männer: $M=3.4$ ), wurden die Stunden für Instandhaltungs- und Reparaturarbeiten zu den Tätigkeiten im Haushalt (Frauen: $M=16.7$, Männer: $M=9.3$ ) hinzugezählt. Daraus ergab sich eine gemeinsame Kategorie der Instandhaltungs-, Reparatur- und Haushaltsarbeiten, die im Folgenden als Hausarbeit bezeichnet wird.

Um ein direktes Maß für die Aufteilung der Erwerbsarbeit, der Hausarbeit sowie der Kinderbetreuung zu erhalten, wurde für die entsprechenden Arbeitsbereiche die vom Mann investierte Stundenzahl zur Gesamtzeit der in der Familie für diesen Arbeitsbereich aufgewendeten Stunden ins Verhältnis gesetzt. Wenn z.B. für die Kinderbetreuung der Mann 10 Stunden, die Frau 30 Stunden und eine weitere Person (z.B. Haushaltshilfe, Kindermädchen, Großeltern) 10 Stunden aufbringen, beträgt der relative Anteil des Mannes an der Kinderbetreuung 10/(10+30+10) $=0.2$. Analog wurde der Anteil des Mannes an der Hausarbeit und der Erwerbsarbeit berechnet (möglicher Range der jeweiligen Variablen zwischen 0 und 1). ${ }^{4}$

Zufriedenheit mit der Arbeitsteilung. Mit je einem Item wurde die Zufriedenheit mit der Aufteilung der Hausarbeit bzw. der Kinderbetreuung erfasst (z.B. „Wie zufrieden sind Sie mit der zwischen Ihnen und Ihrer Partnerin resp. Ihrem Partner bestehenden Aufteilung der Hausarbeit, die es insgesamt zu erledigen gibt?"). Die Einschätzung erfolgte auf einer sechsstufigen Skala $(1=$,gar nicht“ bis $6=$,völlig“ $)$.

3 Sowohl bei Männern als auch bei Frauen lag die selbsteingeschätzte Stundenzahl bei den Haushaltstätigkeiten um etwas mehr als eine Stunde über der vom Partner eingeschätzten Stundenzahl. Der Unterschied war in beiden Fällen signifikant $(t(201) \geq 2.02, p<.05)$. Keinen nennenswerten Unterschied zwischen Selbst- und Fremdeinschätzung gab es bei der Einschätzung der Reparatur- und Instandhaltungsarbeiten $(t(200) \geq 1.39, p>.1)$. Eine gegenläufige Diskrepanz zeigte sich bei der Einschätzung der für Kinderbetreuung benötigten Stundenzahl: für den Beitrag der Männer lag die Fremdeinschätzung der Frauen im Durchschnitt 1.9 Stunden über der Selbsteinschätzung der Männer $(t(200)=2.28, p<.03)$, während umgekehrt für den Beitrag der Frauen die Fremdeinschätzung der Männer 4.9 Stunden unter der Selbsteinschätzung der Frauen lag $(t(201)=3.92, p<.001)$. Mit Ausnahme der Einschätzung der für die Kinderbetreuung aufgewandten Zeit der Männer war somit die Selbsteinschätzung höher als die Fremdeinschätzung. Durch die Mittelung der Selbst- und Fremdeinschätzung werden diese Perspektiveneffekte beseitigt.

4 Der relative Anteil, den andere Personen an der Hausarbeit bzw. Kinderbetreuung übernahmen, betrug im Mittel 6\% bzw. 15\%. 
Partnerschaftszufriedenheit. Die Partnerschaftszufriedenheit wurde mit einer von ursprünglich sieben auf fünf Items reduzierten und leicht modifizierten Version der Relationship Assessment Scale (RAS; Hendrick 1988, deutsche Version von Sander und Böcker 1993) erfasst: 1) „Wie gut wird Ihr Partner bzw. Ihre Partnerin Ihren Bedürfnissen gerecht?“, 2) „In welchem Maß erfüllt diese Partnerschaft Ihre ursprünglichen Erwartungen?“, 3) „Wie zufrieden sind Sie insgesamt gesehen mit Ihrer Partnerschaft?“, 4) „Wie gut ist Ihre Partnerschaft verglichen mit den meisten anderen?“, 5) „Wie zufrieden sind Sie mit Ihrer sexuellen Beziehung?" Das letzte Item wurde hinzugefügt. Die Fragen wurden auf einer sechsstufigen Skala beantwortet $(1=$,gar nicht“" bis $6=$,völlig“ bzw. $1=$ „,sehr schlecht" bis $6=$,sehr gut“). Cronbachs Alpha betrug .86 bei den Frauen und .89 bei den Männern.

Partnerschaftskonflikte. Mit der Aufforderung „Bitte geben Sie an, wie konfliktreich folgende Bereiche in Ihrer Partnerschaft sind“, wurde das Konfliktpotential für die folgenden Bereiche erfasst: finanzielle Angelegenheiten; Freizeitgestaltung; Arbeitsteilung im Haushalt; Sexualität; Beruf/Arbeit; persönliche Eigenheiten und Bedürfnisse; Beziehungen zu Freunden und Verwandten; Arbeitsteilung der Betreuung und Versorgung der Kinder; Erziehungsfragen ${ }^{5}$. Jeder Bereich wurde von beiden Partnern auf einer sechsstufigen Skala von 1 = „gar nicht konfliktreich“ bis 6 = „sehr konfliktreich“ eingeschätzt. Die interne Konsistenz dieser eindimensionalen Skala war zufriedenstellend: Cronbachs Alpha betrug .74 bei den Frauen und .77 bei den Männern.

\section{Statistische Analysen}

$\mathrm{Zu}$ Beginn werden auf Mittelwertsvergleichen und Korrelationen basierende deskriptive Ergebnisse berichtet. Anschließend erfolgt die Überprüfung der Hypothesen im Rahmen von Strukturgleichungsmodellen, welche durch die simultane Erfassung von Angaben beider Partner innovative Auswertungsmöglichkeiten erlauben. Basismodell ist das in Abbildung 1 dargestellte Aktor-Partner-Interdependenz-Modell ${ }^{6}$ (APIM; Kenny et al. 2006), das um Mediatorvariablen erweitert wurde (Ledermann/Bodenmann 2006). Um das Basismodell zu vereinfachen, wurde systematisch geprüft, ob sich Pfade bei Männern und Frauen unterscheiden und ob spezifische direkte Effekte (Pfade $d, e$ oder $g$ ) auf Null fixiert werden können. Dazu wurden in einem ersten Schritt zur Überprüfung von Geschlechtsunterschieden jeweils ein modifiziertes Modell gerechnet, in dem die korrespondierenden Pfade für beide Partner gleichgesetzt wurden (z.B. $f_{f}=f_{m}$ ). Der Fit dieses modifizierten Modells wurde dann mit dem Fit des Basismodells verglichen. Die Fit-Differenz der beiden Modelle folgt einer $\chi^{2}$-Verteilung, die so viele Freiheitsgrade hat, wie Parameter im beschränkten Modell weniger geschätzt werden. Wenn der dem $\chi^{2}$-Wert

5 Der eingesetzte Fragebogen enthielt ebenfalls das Item „Ansichten über Politik, Religion usw.“, das nicht wie alle übrigen Items auf dem gleichen Faktor lud und deshalb nicht in die Skala einbezogen wurde (Meuwly 2007).

6 In den Analysen wurden die absoluten Stunden, die Männer und Frauen für den Beruf und die Hausarbeit bzw. Kinderbetreuung aufwenden, nicht berücksichtigt, obwohl dies von Kenny et al. (2006) gefordert wird, um Haupt- von Interaktionseffekten trennen zu können. Allerdings veränderten sich die hier berichteten Effekte nicht substantiell, wenn die absolute Stundenzahl beider Partner einbezogen wurde. 
zugeordnete $p$-Wert $<.05$ ist, bedeutet dies, dass die korrespondierenden Pfade für Frauen und Männern verschieden sind.

In einem zweiten Schritt wurden dann drei weitere Modelle gerechnet, in denen die direkten Effekte beider Partner auf Null gesetzt waren $\left(d_{f}=d_{m}=0\right.$, bzw. $e_{f}=\mathrm{e}_{m}=0$ bzw. $g_{f}=g_{m}=0$ ). Der Fit dieser Modelle wurde dann wieder mit dem Basismodell bzw. wenn keine Geschlechtseffekte in den geprüften Pfaden vorlagen, mit dem modifizierten Modell verglichen. Zum Schluss berechneten wir ein vereinfachtes Endmodell, in welchem sämtliche Pfade für beide Partner gleichgesetzt und direkte Pfade beider Partner auf Null fixiert wurden, wenn die vorangegangene Überprüfung keine signifikante Verschlechterung des Modellfits $(p<.05)$ ergeben hatte. Von diesem Endmodell berichteten wir die Regressionskoeffizienten.

Um zu prüfen, ob der Zusammenhang zwischen der Zufriedenheit mit der Arbeitsteilung und der Partnerschaftszufriedenheit durch die erlebten Konflikte mediiert wird, berechneten wir jeweils den indirekten Effekt. Bei den Frauen liegt ein Mediationseffekt vor, wenn sowohl die einzelnen Pfade $f_{f}$ und $i_{f}$ wie auch das Produkt der Pfade $f_{f} * i_{f}$, d.h. der gesamte indirekte Effekt, signifikant sind. Bei den Männern müssen die korrespondierenden Koeffizienten $f_{m}, i_{m}$ und $f_{m}{ }^{*} i_{m}$ signifikant sein (Ledermann/Bodenmann 2006; Shrout/Bolger 2002). Falls bei Berücksichtigung einer Mediationsvariable der direkte Effekt (im Beispiel $g_{f}$ bzw. $g_{m}$ ) signifikant ist, handelt es sich um eine partielle Mediation, ansonsten um eine vollständige Mediation.

Die Analysen wurden mit Mplus 5 (Muthén/Muthén 2007) gerechnet. Dieses Programm schätzt Standardfehler für die indirekten Effekte und erleichtert dadurch die Signifikanzprüfung. Da die Daten nicht normalverteilt waren, wurde zur Berechnung der Standardfehler Bootstrap-Analysen durchgeführt (mit 500 bootstrap-samples).

\section{Ergebnisse}

\section{Deskriptive Analysen}

Bei den untersuchten Paaren waren Erwerbs- und Familienarbeit überwiegend traditionell aufgeteilt. Die Männer waren 17.5 Stunden pro Woche länger mit beruflichen Tätigkeiten (inklusive Arbeitsweg) beschäftigt als ihre Frauen (M (SD): 45.6 (11.4) vs. 28.1 (8.4), $t(206)=17.22, p<.001)$, während sie deutlich weniger Zeit als diese mit Hausarbeit $(12.6(6.4)$ vs. $18.9(7.9), t(206)=11.12, p<.001)$ und Kinderbetreuung $(23.7(11.0)$ vs. $38.5(13.1), t(206)=15.04, p<.001)$ verbrachten. Zählt man die für Erwerbsarbeit, Hausarbeit und Kinderbetreuung geleisteten Stunden zusammen, dann war der gesamte Arbeitsaufwand der Männer etwa 3.5 Stunden pro Woche geringer als der ihrer Frauen (82.0 (13.4) vs. 85.5 (15.4), $t(206)=3.99, p<.001)$. Männer waren mit der Aufteilung der Hausarbeit $(t(202)=5.40, p<.001)$ und der Aufteilung der Kinderbetreuung zufriedener als Frauen $(t(202)=2.22, p=.028)$ (vgl. Tab. 1). Im wahrgenommenen Konfliktpotential und in der Partnerschaftszufriedenheit gab es dagegen keine Geschlechtsunterschiede $(t(206) \leq 1.39, p>.1)$.

Aus den Korrelationen in Tabelle 1 geht hervor, dass der Anteil der Männer an der Hausarbeit und Kinderbetreuung umso geringer war, je mehr Zeit sie im Verhältnis zu ih- 
ren Frauen für den Beruf benötigten. Interessant ist, dass bei Männern, die sich stärker an der Hausarbeit und Kinderbetreuung beteiligten, nicht nur ihre Partnerinnen mit der Arbeitsteilung zufriedener waren, sondern auch sie selbst. Ferner war die Zufriedenheit mit der Aufteilung der Kinderbetreuung - nicht aber die Zufriedenheit mit der Aufteilung der Hausarbeit - bei beiden Partnern umso höher, je geringer der relative Anteil an Zeit war, den Männer für den Beruf investierten.

Tabelle 1: Korrelationen, Mittelwerte und Standardabweichungen der Variablen $(N=207$ Paare $)$

\begin{tabular}{|c|c|c|c|c|c|c|c|c|c|c|c|c|}
\hline & & 1 & 2 & 3 & 4 & 5 & 6 & 7 & 8 & 9 & 10 & 11 \\
\hline 1 & $\begin{array}{l}\text { Relativer Anteil } \\
\text { der Männer an } \\
\text { Erwerbsarbeit }\end{array}$ & & & & & & & & & & & \\
\hline 2 & $\begin{array}{l}\text { Relativer Anteil } \\
\text { der Männer HA }\end{array}$ & $-.68^{* * *}$ & & & & & & & & & & \\
\hline 3 & $\begin{array}{l}\text { Relativer Anteil } \\
\text { der Männer KB }\end{array}$ & $-.64^{* * *}$ & $.57^{\star \star *}$ & & & & & & & & & \\
\hline 4 & $\begin{array}{l}\text { Zufriedenheit mit } \\
\text { Aufteilung HA } \\
\text { Frauen }\end{array}$ & -.11 & $.36^{* * *}$ & $.20^{* *}$ & & & & & & & & \\
\hline 5 & $\begin{array}{l}\text { Zufriedenheit mit } \\
\text { Aufteilung HA } \\
\text { Männer }\end{array}$ & -.14 & $.19^{* *}$ & $.14^{*}$ & $.22^{* *}$ & & & & & & & \\
\hline 6 & $\begin{array}{l}\text { Zufriedenheit mit } \\
\text { Aufteilung KB } \\
\text { Frauen }\end{array}$ & $-.18^{* *}$ & $.23^{* *}$ & $.27^{* * *}$ & $.52^{\star \star \star}$ & $.19^{\star *}$ & & & & & & \\
\hline 7 & $\begin{array}{l}\text { Zufriedenheit mit } \\
\text { Aufteilung KB } \\
\text { Männer }\end{array}$ & $-.29^{* \star *}$ & $.33^{* \star *}$ & $.23^{* *}$ & $.31^{* \star *}$ & $.54^{* * *}$ & $.33^{* \star *}$ & & & & & \\
\hline 8 & $\begin{array}{l}\text { Partnerschafts- } \\
\text { konflikte Frauen }\end{array}$ & .03 & $-.16^{*}$ & .00 & $-.45^{* * *}$ & $-.20^{* *}$ & $-.39^{* * *}$ & -.13 & & & & \\
\hline 9 & $\begin{array}{l}\text { Partnerschafts- } \\
\text { konflikte Männer }\end{array}$ & .13 & $-.20^{\star *}$ & -.07 & $-.28^{* * *}$ & $-.23^{\star *}$ & $-.19^{* *}$ & $-.32^{* * *}$ & $.46^{* * *}$ & & & \\
\hline 10 & $\begin{array}{l}\text { Partnerschafts- } \\
\text { zufriedenheit } \\
\text { Frauen }\end{array}$ & -.01 & .07 & . 06 & $.48^{* * *}$ & $.16^{*}$ & $.36^{* * *}$ & $.18^{*}$ & $-.59^{* * *}$ & $-.40^{* *}$ & & \\
\hline 11 & $\begin{array}{l}\text { Partnerschafts- } \\
\text { zufriedenheit } \\
\text { Männer }\end{array}$ & .00 & .02 & -.05 & $.20^{* *}$ & $.19^{* *}$ & $.18^{*}$ & $.30^{* * \star}$ & $-.37^{\star \star *}$ & $-.61^{* * *}$ & $.59^{* \star *}$ & \\
\hline & Mittelwert & .62 & .38 & .32 & 4.33 & 4.83 & 4.72 & 4.90 & 2.57 & 2.54 & 4.85 & 4.77 \\
\hline & $(S D)$ & .10 & .12 & .11 & 1.21 & .94 & 1.16 & 1.02 & .76 & .75 & .79 & .88 \\
\hline
\end{tabular}

Anmerkung: $\mathrm{HA}=$ Hausarbeit; $\mathrm{KB}=$ Kinderbetreuung. ${ }^{*} p<.05 ;{ }^{* *} p<.01 ; * * * p .00$

Männer und Frauen, die zufriedener waren mit der Aufteilung der Hausarbeit, waren auch zufriedener mit der Aufteilung der Kinderbetreuung, und ihre Partner waren mit der Arbeitsteilung in beiden Bereichen zufriedener. Sowohl die eigene Zufriedenheit mit der familiären Arbeitsteilung als auch die des Partners ging mit weniger wahrgenommenen Konflikten und einer höheren Partnerschaftszufriedenheit einher. Dagegen war weder der 
Anteil, den Männer an der Erwerbsarbeit hatten, noch der Anteil, den sie an der Kinderbetreuung hatten, mit den wahrgenommenen Konflikten oder der Partnerschaftszufriedenheit assoziiert. Lediglich die Aufteilung der Hausarbeit war schwach mit den von beiden Partnern wahrgenommenen Konflikten assoziiert, jedoch nicht mit der Partnerschaftszufriedenheit. Wie erwartet war die Partnerschaftszufriedenheit umso höher, je geringer die selbst berichteten Konflikte wie auch die berichteten Konflikte des Partners waren. Insgesamt passt das Muster an Korrelationen gut zu den in den Hypothesen formulierten Vorhersagen.

\section{Ergebnisse zur Aufteilung der Hausarbeit}

Vereinfachung des Modells

Das Basismodell für die Aufteilung der Hausarbeit (vgl. Abb. 1) war sehr gut mit den Daten vereinbar $\left(\chi^{2}(6)=2.27, p=.894, C F I=1, R M S E A<.001\right.$, bootstrap- $\left.p=.976\right)$. Die Überprüfung von Geschlechtsunterschieden ergab, dass folgende Effekte sich nicht zwischen Frauen und Männern unterschieden $\left(\Delta \chi^{2}(1) \leq 1.90, p \geq .168\right)$ : die direkten Effekte des Anteils der Männer an der Hausarbeit auf die Partnerschaftskonflikte $\left(d_{f}=d_{f}\right)$, die direkten Effekte des Anteils der Männer an der Hausarbeit auf die Partnerschaftszufriedenheit $\left(e_{f}=e_{m}\right)$, die Partnereffekte der Zufriedenheit mit der Aufteilung der Hausarbeit auf die berichteten Konflikte $\left(h_{m f}=h_{f m}\right)$ sowie die Partnereffekte der berichteten Konflikte auf die Partnerschaftszufriedenheit $\left(j_{m f}=j_{f m}\right)$. In den übrigen Pfaden $(b, c, f, g$ und $i)$ gab es dagegen signifikante Unterschiede zwischen Frauen und Männern $\left(\Delta \chi^{2}(1) \geq 3.90, p \leq .049\right)$.

Die weitere Überprüfung ergab, dass die direkten Effekte des Anteils, den Männer an der Hausarbeit leisten, auf die Partnerschaftskonflikte $\left(d_{f}\right.$ und $\left.d_{m}\right)$ Null waren $\left(\Delta \chi^{2}(1)=\right.$ $0.51, p=.476)$. Von Null verschieden waren dagegen die direkten Effekte des Anteils der Männer an der Hausarbeit auf die Partnerschaftszufriedenheit $\left(e_{f}\right.$ und $\left.e_{m}\right)\left(\Delta \chi^{2}(1)=6.34, p\right.$ $=.012$ ), sowie die direkten Effekte der Zufriedenheit mit der Aufteilung der Hausarbeit auf die Partnerschaftszufriedenheit $\left(g_{f}\right.$ und $\left.g_{m}\right)\left(\Delta \chi^{2}(2)=26.77, p \leq .001\right)$.

Abschließend berechneten wir ein vereinfachtes Endmodell, aus dem wir die Pfade $d_{f}$ und $d_{m}$ entfernten und die Pfade für Männer und Frauen gleich setzten, die sich nicht signifikant unterschieden. Der Fit dieses Modells war nicht signifikant schlechter als der Fit des Basismodells $\left(\Delta \chi^{2}(5)=3.47, p=.629\right)$ und zeigt an, dass auch das vereinfachte Modell sehr gut zu den beobachteten Daten passte $\left(\chi^{2}(11)=5.73, p=.891, C F I=1, R M S E A\right.$ $<.001$, bootstrap $-p=.952$ ). 
Abbildung 1: Theoretische Modellierung der Zusammenhänge im Rahmen eines um Mediatorvariablen erweiterten Aktor-Partner-Interdependenz-Modells

$g_{f}$

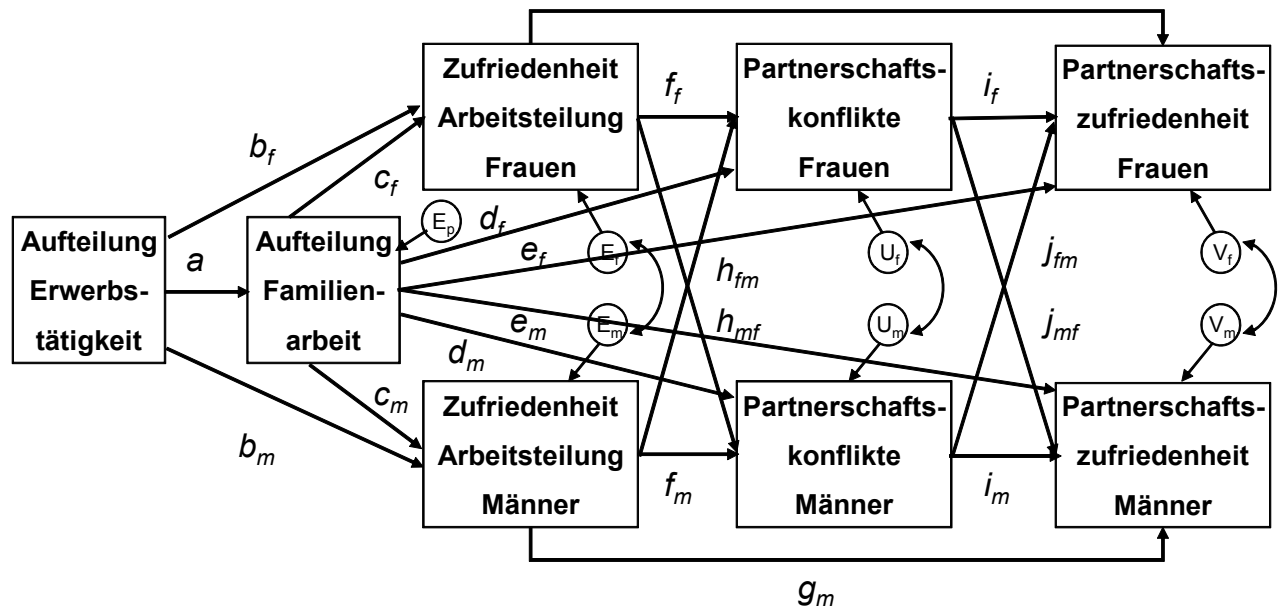

Anmerkung: Dieses Modell wird für die Hausarbeit und die Kinderbetreuung getrennt berechnet, d.h. die Aufteilung der Familienarbeit und die Zufriedenheit mit der Arbeitsteilung beziehen sich jeweils auf den entsprechenden Bereich (Hausarbeit bzw. Kinderbetreuung).

Interpretation der Koeffizienten des Modells

Die Koeffizienten des vereinfachten Endmodells werden in Tabelle 2 berichtet. Sie lassen sich folgendermaßen interpretieren: Je größer der Anteil der Männer an der Erwerbsarbeit war, desto geringer war ihr relativer Beitrag an der Hausarbeit $(\beta a=-.68)$. Frauen waren umso zufriedener mit der Aufteilung der Hausarbeit, je größer der Anteil ihres Partners an der Erwerbstätigkeit war $\left(\beta b_{f}=.25\right)$ und je mehr sich ihr Partner unabhängig davon für die Hausarbeit engagierte $\left(\beta c_{f}=.52\right)$. Bei Männern gab es diese Zusammenhänge nicht ( $\beta$ $b_{m}$ und $\beta c_{m}$ n.s.).

Beide Partner gaben an, mehr Konflikte zu erleben, wenn sie selbst unzufrieden mit der Aufteilung der Hausarbeit waren. Dieser Aktoreffekt war, wie von H-1.1 vorhergesagt, bei Frauen stärker ausgeprägt als bei Männern $\left(\beta f_{f}=-.41\right.$ vs. $\beta f_{m}=-.21 ; \chi^{2}(1)=$ $3.91, p=.048)$. Darüber hinaus hatte auch die Zufriedenheit des Partners mit der Aufteilung der Hausarbeit einen Effekt auf die berichteten Konflikte $(\beta h \leq-.15)^{7}$. Je zufriedener also Männer und Frauen mit der Arbeitsteilung waren, desto weniger Partnerschaftskonflikte berichtete ihr Partner (Bewährung H-1.2a und b).

Der Zusammenhang zwischen dem Anteil des Mannes an der Hausarbeit und den Partnerschaftskonflikten wurde für beide Partner vollständig über die Zufriedenheit mit der Arbeitsteilung vermittelt (die Summe der indirekten Effekte waren signifikant [Frau-

7 Da die unstandardisierten Koeffizienten gleichgesetzt waren, sind Unterschiede in den standardisierten Koeffizienten auf Unterschiede in den Varianzen zurückzuführen. 
en: $\Lambda=-.24, \mathrm{z}=-4.84, p<.00$; Männer: $\Lambda=-.14, \mathrm{z}=-3.76, p<.001]$, die direkten Effekte $d_{f}$ und $d_{m}$ dagegen Null), was H-1.3a und b bestätigt. Die Partnerschaftszufriedenheit war in Übereinstimmung mit H-2.1 bei beiden Partnern deutlich höher, wenn sie selbst weniger Konflikte berichteten, wobei der Effekt bei den Männern ausgeprägter war, als bei den Frauen $\left(\beta i_{f}=-.42\right.$ vs. $\left.\beta i_{m}=-.55: \chi^{2}(1)=4.45, p=.035\right)$. Unabhängig davon war die Partnerschaftszufriedenheit ebenfalls höher, wenn der Partner bzw. die Partnerin weniger Konflikte berichtete $(\beta j \leq-.14)$, was $\mathrm{H}-2.2 \mathrm{a}$ und $\mathrm{H}-2.2 \mathrm{~b}$ bestätigt. Bei beiden Partnern war die Partnerschaftszufriedenheit umso geringer, je höher der Beitrag der Männer an der Hausarbeit war $(\beta e \leq-.11)$. Die negative Richtung dieses Zusammenhangs ist zumindest bei den Frauen unerwartet. $\mathrm{Zu}$ beachten ist jedoch, dass es sich um einen Nettoeffekt handelt, d.h. der Einfluss, den die wahrgenommenen Konflikte beider Partner sowie die Zufriedenheit mit der Aufteilung der Hausarbeit auf die Partnerschaftszufriedenheit haben, ist kontrolliert.

Tabelle 2: Modellparameter für die Hausarbeit und Kinderbetreuung $(N=207$ Paare $)$

\begin{tabular}{|c|c|c|c|c|c|c|c|c|c|}
\hline & & \multicolumn{4}{|c|}{ Hausarbeit } & \multicolumn{4}{|c|}{ Kinderbetreuung } \\
\hline & & $\mathrm{R}^{2}$ & $\mathrm{~B}$ & (SE) & $\beta$ & $\mathrm{R}^{2}$ & B & (SE) & $\beta$ \\
\hline \multicolumn{2}{|r|}{ Anteil der Männer an der Familienarbeit (aV) } & .46 & & & & .41 & & & \\
\hline$a$ & Anteil der Männer an der Erwerbsarbeit & & -.86 & .07 & $-.68^{* * *}$ & & -.73 & .06 & $-.64^{\star * *}$ \\
\hline \multicolumn{2}{|r|}{ Zufriedenheit der Frauen mit der Arbeitsteilung (aV) } & .16 & & & & .06 & & & \\
\hline$b_{f}$ & Anteil der Männer an der Erwerbsarbeit & & 3.12 & 1.14 & $.25^{\star *}$ & & -1.63 & 1.07 & -.14 \\
\hline$c_{f}$ & Anteil der Männer an der Familienarbeit & & 5.27 & .86 & $.52^{\star \star \star}$ & & 1.52 & .70 & $.14^{*}$ \\
\hline \multicolumn{2}{|r|}{ Zufriedenheit der Männer mit der Arbeitsteilung (aV) } & .04 & & & & .08 & & & \\
\hline$b_{m}$ & Anteil der Männer an der Erwerbsarbeit & & -.11 & .88 & -.01 & & -1.63 & 1.07 & -.15 \\
\hline$c_{m}$ & Anteil der Männer an der Familienarbeit & & 1.40 & .83 & .18 & & 1.52 & .70 & $.16^{*}$ \\
\hline \multicolumn{2}{|r|}{ Von Frauen berichtete Partnerschaftskonflikte (aV) } & .21 & & & & .14 & & & \\
\hline$f_{f}$ & Zufriedenheit der Frauen mit der Arbeitsteilung & & -.26 & .04 & $-.41^{* * *}$ & & -.23 & .03 & $-.36^{* * *}$ \\
\hline$h_{f m}$ & Zufriedenheit der Männer mit der Arbeitsteilung & & -.12 & .03 & $-.15^{* * *}$ & & -.03 & .03 & -.04 \\
\hline \multicolumn{2}{|r|}{ Von Männern berichtete Partnerschaftskonflikte (aV) } & .10 & & & & .11 & & & \\
\hline$f_{m}$ & Zufriedenheit der Männer mit der Arbeitsteilung & & -.17 & .05 & $-.21^{\star *}$ & & -.23 & .03 & $-.32^{* \star *}$ \\
\hline$h_{m f}$ & Zufriedenheit der Frauen mit der Arbeitsteilung & & -.12 & .03 & $-.19^{* * *}$ & & -.03 & .03 & -.05 \\
\hline \multicolumn{2}{|r|}{ Partnerschaftszufriedenheit der Frau (aV) } & .43 & & & & \\
\hline$e_{f}$ & Anteil der Männer an der Familienarbeit & & -.83 & .34 & $-.13^{*}$ & \multicolumn{4}{|c|}{ (auf Null fixiert) } \\
\hline$g_{f}$ & Zufriedenheit der Frauen mit der Arbeitsteilung & & .18 & .04 & $.28^{* * *}$ & & .10 & .03 & $.14^{* *}$ \\
\hline$i_{f}$ & Von Frauen berichtete Partnerschaftskonflikte & & -.44 & .06 & $-.42^{* * *}$ & & -.53 & .04 & $-.50^{* * *}$ \\
\hline & Von Männern berichtete Partnerschaftskonflikte & & -.16 & .04 & $-.15^{\star * *}$ & & -.16 & .04 & $-.15^{* * *}$ \\
\hline \multicolumn{2}{|r|}{ Partnerschaftszufriedenheit der Männer (aV) } & .40 & & & & \multicolumn{4}{|l|}{.36} \\
\hline$e_{m}$ & Anteil der Männer an der Familienarbeit & & -.83 & .34 & $-.11^{*}$ & \multicolumn{4}{|c|}{ (auf Null fixiert) } \\
\hline$g_{m}$ & Zufriedenheit der Männer mit der Arbeitsteilung & & .05 & .05 & .05 & & .10 & .03 & $.12^{* *}$ \\
\hline$i_{m}$ & Von Männern berichtete Partnerschaftskonflikte & & -.64 & .06 & $-.55^{\star * *}$ & & -.53 & .04 & $-.47^{\star * *}$ \\
\hline & Von Frauen berichtete Partnerschaftskonflikte & & -.16 & .04 & $-.14^{* \star *}$ & & -.16 & .04 & $-.14^{* * *}$ \\
\hline
\end{tabular}

Im Modell für die Hausarbeit sind die Pfade e, h und j der Frauen und Männer gleichgesetzt, im Modell für die Kinderbetreuung dagegen alle Pfade.

Die Pfade $\mathrm{d}_{f}$ und $\mathrm{d}_{m}$ in Abb. 1 sind in beiden Modellen auf Null fixiert. ${ }^{*} \mathrm{p}<.05 ;{ }^{* *} \mathrm{p}<.01 ; * * * \mathrm{p}<.001$ 
Überprüfung der Mediatoreffekte

Die Mediatorhypothese H-3, die besagt, dass die Zufriedenheit mit der Arbeitsteilung sich indirekt über das wahrgenommene Konfliktpotential auf die Partnerschaftszufriedenheit auswirkt, konnte bestätigt werden. Bei beiden Partnern waren sowohl die einzelnen Pfade $\left(f_{f}\right.$ und $i_{f}$, sowie $f_{m}$ und $i_{m}$ ) wie auch deren Produkt signifikant (Frauen: $\beta f_{f} * i_{f}=.17, z=$ 4.68, $p<.001$; Männer: $\left.\beta f_{m}{ }^{*} i_{m}=.12, z=3.37, p<.001\right)$. Neben der soeben beschriebenen Mediation durch Aktoreffekte bestand auch eine signifikante Mediation durch Partnereffekte. So ging z.B. eine höhere Zufriedenheit der Frauen mit der Arbeitsteilung damit einher, dass der Partner weniger Konflikte berichtete (Partnereffekt: $\beta h_{m f}=-.19$ ), was sich wiederum günstig auf die Partnerschaftszufriedenheit der Frauen auswirkte (Partnereffekt: $\left.\beta j_{f m}=-.15\right)$. Aus dem Produkt der beiden Partnereffekte ergibt sich der PartnerMediatoreffekt, der zwar sehr klein aber dennoch signifikant war $\left(\beta h_{m f} * j_{f m}=.03, \mathrm{z}=\right.$ $2.83, p=.005$ ). Der korrespondierende Partner-Mediatoreffekt für die Männer, der erfasst, welchen Anteil das Konflikterleben der Frauen am Zusammenhang zwischen der von den Männern berichteten Zufriedenheit mit der Arbeitsteilung und ihrer Partnerschaftszufriedenheit hat, war vergleichbar $\left(\beta h_{f m}{ }^{*} j_{m f}=.02, \mathrm{z}=2.63, p=.008\right)$.

Bei den Frauen war die Partnerschaftszufriedenheit auch nach Kontrolle der berichteten Partnerschaftskonflikte umso höher, je zufriedener sie mit der Aufteilung der Hausarbeit waren $\left(\beta g_{f}=.28\right)$. Bei den Männern gab es dagegen keinen direkten Effekt der Zufriedenheit mit der Arbeitsteilung auf die Partnerschaftszufriedenheit $\left(\beta g_{m}\right.$, n.s.). Somit wurde der Zusammenhang zwischen der Zufriedenheit mit der Aufteilung der Hausarbeit und der Partnerschaftszufriedenheit bei den Frauen partiell und bei den Männern vollständig durch die wahrgenommenen Konflikte mediiert.

Gemäß H-4a und H-4b war der gesamte indirekte Effekt der Aufteilung der Hausarbeit auf die Partnerschaftszufriedenheit, der über die Zufriedenheit mit der Arbeitsteilung läuft, sowohl bei den Frauen $\left(\Delta \chi^{2}(1)=26.09, p<.001\right)$ wie auch bei den Männern $\left(\Delta \chi^{2}(1)\right.$ $=16.13, p<.001)$ größer als die Summe der Effekte, die nicht über die Zufriedenheit mit der Arbeitsteilung laufen (die Summe dieser Effekte reduzierte sich hier auf den direkten Effekt von $e_{m}$ bzw. $e_{f}$, da $d_{f}$ und $d_{m}$ auf Null fixiert waren). Die Summe der indirekten Effekte der Arbeitsteilung der Hausarbeit auf die Partnerschaftszufriedenheit betrug für die Frauen: $\Lambda=.27, \mathrm{z}=5.13, p<.001$; für die Männer: $\Lambda=.12, \mathrm{z}=3.71, p<.001$.

\section{Ergebnisse zur Aufteilung der Kinderbetreuung}

Vereinfachung des Modells

Auch bei der Modellierung der Aufteilung der Kinderbetreuung war das Basismodell sehr gut mit den Daten vereinbar $\left(\chi^{2}(6)=2.49, p=.870, C F I=1, R M S E A<.001\right.$, bootstrap- $p$ $=.956$ ). Lediglich die direkten Pfade von der Aufteilung der Kinderbetreuung zur Partnerschaftszufriedenheit $\left(e_{m}\right.$ und $\left.e_{f}\right)$ waren für Männer und Frauen signifikant verschieden $\left(\Delta \chi^{2}(1)=4.65, p=.031\right)$, alle anderen Pfade $(b, c, d, f, g, h, i$ und $j)$ konnten dagegen für Männer und Frauen gleich gesetzt werden $\left(\Delta \chi^{2}(1) \leq 3.61, p \geq .058\right)$. Allerdings waren die direkten Effekte $e_{f}$ und $e_{m}$ nicht signifikant von Null verschieden $\left(\Delta \chi^{2}(2)=3.33, p=.189\right)$, ebenso wenig wie die direkten Effekte von der Aufteilung der Kinderbetreuung auf die 
Partnerschaftskonflikte $\left(d_{f}=d_{m}=0\right)\left(\Delta \chi^{2}(1)=1.89, p=.169\right)$. Nur die direkten Effekte von der Zufriedenheit mit der Aufteilung der Kinderbetreuung auf die Partnerschaftszufriedenheit $\left(g_{f}\right.$ und $\left.g_{m}\right)$ waren signifikant größer Null $\left(\Delta \chi^{2}(1)=12.17, p \leq .001\right)$.

Das vereinfachte Modell aus dem die Pfade $d_{f}, d_{m}, e_{f}$ und $e_{m}$ entfernt wurden und sämtliche Pfade für Männer und Frauen gleichgesetzt waren, hatte keinen schlechteren Fit als das Basismodell $\left(\Delta \chi^{2}(11)=15.77, p=.150\right)$ und passte sehr gut $\mathrm{zu}$ den beobachteten Daten $\left(\chi^{2}(17)=18.26, p=.373, C F I=.998, R M S E A=.019\right.$, bootstrap $\left.-p=.594\right)$.

Interpretation der Koeffizienten des Modells

Die Männer beteiligten sich umso weniger an der Kinderbetreuung, je mehr sie zur Erwerbsarbeit beitrugen ( $\beta a=-.64)$ (vgl. Tab. 2). Beide Partner waren mit der Aufteilung der Kinderbetreuung zufriedener, wenn sich der Mann stärker an der Kinderbetreuung beteiligte $(\beta c \geq .14)$, der Anteil der Männer an der Erwerbsarbeit hatte darauf keinen Einfluss $(\beta b$, n.s.). Beide Partner gaben an, mehr Konflikte zu erleben, wenn sie selbst unzufrieden mit der Aufteilung der Kinderbetreuung waren $(\beta f \leq-.32)$. Entgegen den Erwartungen war jedoch dieser Aktoreffekt bei Frauen nicht höher als bei Männern $\left(\chi^{2}(1)=\right.$ $0.57, p=.449$, Widerlegung H-1.1) und es gab keine korrespondierenden Partnereffekte ( $\beta$ h n.s., Widerlegung H-1.2a und b). Wie bereits im Modell für die Hausarbeit konnten die direkten Effekte der Aufteilung der Kinderbetreuung auf die Partnerschafskonflikte auf Null gesetzt werden, d.h. im Einklang mit H-1.3 bestand für beide Partner lediglich ein indirekter Zusammenhang zwischen der Arbeitsteilung der Kinderbetreuung und den berichteten Partnerschaftskonflikten, welcher über die Zufriedenheit mit der Arbeitsteilung verlief. Die Summe der indirekten Effekte war signifikant $(\Lambda=-.06 \mathrm{z} \geq-2.14, p<$ $.05)$. Die Partnerschaftszufriedenheit beider Partner war umso geringer, je mehr Partnerschaftskonflikte sie selber und auch ihr Partner wahrgenommen hatten (Aktoreffekte: $\beta i$ $\leq-.47$, Partnereffekte: $\beta j \leq-.14$ ), was H-2.1 und H-2.2 bestätigt. Unabhängig davon war die Partnerschaftszufriedenheit bei beiden Partnern umso höher, je zufriedener sie mit der Aufteilung der Kinderbetreuung waren $(\beta g \geq .12)$.

\section{Überprüfung der Mediatoreffekte}

Bei beiden Partnern waren sowohl die einzelnen Aktoreffekte ( $f$ und $i$ ), wie auch deren Produkt signifikant $(\beta f * i \geq .15, z \geq 6.44, p<.001)$. Dies bestätigt Mediatorhypothese $\mathrm{H}$ 3 , die besagt, dass die Zufriedenheit mit der Aufteilung der Kinderbetreuung sich über das wahrgenommene Konfliktpotential auf die Partnerschaftszufriedenheit auswirkt. Die Partner-Mediatoreffekte waren dagegen nicht signifikant $\left(\beta h^{*} j=.01, z \leq 0.96, p \geq .335\right)$. Da auch die direkten Effekte $\left(g_{f}\right.$ resp. $\left.g_{m}\right)$ signifikant waren, liegt für beide Partner eine partielle Mediation vor.

Der gesamte indirekte Effekt den die Aufteilung der Kinderbetreuung auf die Partnerschaftszufriedenheit hat, war klein, aber signifikant $(\Lambda=.06, \mathrm{z} \geq 2.12, p<.05)$. Da die Summe der Effekte, die nicht über die Zufriedenheit mit der Aufteilung der Kinderbetreuung vermittelt sind, Null war, bestätigt dieses Ergebnis H-4, die besagt, dass die subjektive Bewertung der Arbeitsteilung bedeutender für die Partnerschaftszufriedenheit ist als die tatsächliche Aufteilung der Kinderbetreuung. 


\section{Diskussion}

Ziel der vorliegenden Studie war es, zu untersuchen, wie sich die Aufteilung von Hausarbeit und Kinderbetreuung auf die Partnerschaftszufriedenheit von Eltern, die beide berufstätig sind, auswirkt. Unsere zentrale Erwartung war, dass weniger der Anteil der geleisteten Stunden, sondern vielmehr die subjektive Bewertung der bestehenden Aufteilung die Partnerschaftszufriedenheit beider Partner beeinflusst. Dabei gingen wir davon aus, dass Männer und Frauen, die mit der Aufteilung der Familienarbeit zufrieden sind, weniger Partnerschaftskonflikte erleben und dadurch wiederum zufriedener mit ihrer Partnerschaft sind. Um diese Erwartungen zu überprüfen, wurden Einschätzungen von beiden Partnern erhoben und in einem erweiterten Aktor-Partner-Interdependenzmodell berechnet. Sowohl für die Aufteilung der Hausarbeit als auch für die Aufteilung der Kinderbetreuung, die den Empfehlungen von Ishii-Kuntz und Coltrane (1992; Coltrane 2000) folgend, getrennt betrachtet wurden, konnten die zentralen Erwartungen bestätigt werden.

In den Korrelationsanalysen zeigte sich kein Zusammenhang zwischen der Partnerschaftszufriedenheit und der tatsächlichen Aufteilung der Kinderbetreuung bzw. Hausarbeit. Dagegen war im berechneten Aktor-Partner-Modell der direkte Effekt der Aufteilung der Hausarbeit auf die Partnerschaftszufriedenheit (der für beide Partner gleichgesetzt werden konnte) signifikant, der Effekt war jedoch klein. Inhaltlich besagt dieser Partialeffekt, dass die vom Einfluss der erlebten Konflikte (sowie der Zufriedenheit mit der Arbeitsteilung) bereinigte Partnerschaftszufriedenheit der Männer, aber auch die der Frauen tendenziell geringer ist, wenn die Männer einen größeren Anteil an der Hausarbeit übernehmen. Dieser negative Effekt könnte durch Geschlechtsrollenideologien bestimmt sein: durch die Sozialisation haben beide Partner gelernt, dass die Frau hauptsächlich für die Hausarbeit zuständig ist. Trotz einer erhöhten Zufriedenheit mit der Arbeitsteilung könnten Bewertungsprozesse, wie „bin ich eine gute Frau resp. ein guter Mann, wenn sich der Mann vermehrt für die Hausarbeit engagiert" diesen direkten negativen Zusammenhang als Diskrepanz zum positiven indirekten Effekt erklären.

Weitere Analysen zeigen, dass die tatsächliche Aufteilung der Hausarbeit bzw. Kinderbetreuung hauptsächlich einen über die Zufriedenheit mit dieser Aufteilung vermittelten indirekten Effekt auf die wahrgenommenen Partnerschaftskonflikte (Bewährung H1.3) und die Partnerschaftszufriedenheit (Bewährung H-4) hatte. Mit der Partnerschaftszufriedenheit deutlich assoziiert war die Zufriedenheit mit der Aufteilung der Hausarbeit und Kinderbetreuung. Insgesamt zeigen unsere Ergebnisse, dass nicht die tatsächliche Aufteilung der familiären Arbeit, sondern deren Bewertung durch die Partner entscheidend ist für das Klima in der Partnerschaft. Sie stimmen überein mit den Ergebnissen anderer Studien, in denen die Zufriedenheit mit der Aufteilung der familiären Arbeit bzw. die Wahrnehmung der Gerechtigkeit der Aufteilung untersucht wurde (z.B. Blair 1998; Mikula et al. 2008; Voydanoff/Donnelly 1999; Yogev/Brett 1985).

Darüber hinaus konnten wir belegen, dass der Zusammenhang zwischen der Zufriedenheit mit der Aufteilung der familiären Arbeit und der Partnerschaftszufriedenheit zumindest partiell über die Konflikthäufigkeit mediiert wurde (Bewährung H-3). Eine höhere Zufriedenheit mit der Aufteilung der familiären Arbeit reduzierte die wahrgenommenen Partnerschaftskonflikte, was wiederum einen positiven Effekt auf die eigene Partnerschaftszufriedenheit wie auch die des Partners hatte (Bewährung H-2.1 und 2.2). 
Unterschiede zwischen Hausarbeit und Kinderbetreuung

Vergleicht man beide Tätigkeitsbereiche, fällt auf, dass es nur bezüglich der Hausarbeit Geschlechtsunterschiede im Muster der Zusammenhänge gab. Im Hinblick auf die Kinderbetreuung waren die Effekte für Männer und Frauen dagegen gleich. Im Gegensatz zur Hausarbeit und entgegen unserer Erwartung (Widerlegung H-1.1 und 1.2 für die Kinderbetreuung) beeinflusste die Zufriedenheit mit der Aufteilung der Kinderbetreuung lediglich die selbst berichteten Konflikte, nicht aber die vom Partner berichteten Konflikte (kein Partnereffekt). Dies hängt möglicherweise damit zusammen, dass die Aufteilung der Kinderbetreuung weniger häufig Konfliktthema in der Partnerschaft war als die Aufteilung der Hausarbeit (dies geht aus den entsprechenden Einzelitems der Konfliktskala hervor).

Der Anteil, den Männer an der Kinderbetreuung und an der Hausarbeit übernahmen, korrelierte hoch miteinander. Das heißt: Männer, die sich stärker an der familiären Arbeit beteiligten, taten dies sowohl bei der Kinderbetreuung als auch bei der Hausarbeit. Wie viel sie für Familienarbeit investierten, hing dabei maßgeblich von der Aufteilung der Berufsarbeit ab. Die Zufriedenheit mit der Aufteilung in beiden Bereichen war ebenfalls hoch korreliert. Bemerkenswert ist, dass insbesondere die Männer eine deutlich höhere Zufriedenheit mit der Aufteilung der Kinderbetreuung berichteten, wenn sie selbst weniger für den Beruf arbeiteten. Diese Ergebnisse zeigen, dass die Aufteilung der beruflichen Arbeit zwischen den Partnern eng mit der Aufteilung der familiären Arbeit, aber auch mit der Bewertung dieser Aufteilung assoziiert ist.

Trotz Übereinstimmungen zwischen Kinderbetreuung und Hausarbeit ist es wichtig, dass beide Tätigkeitsbereiche separat erfasst und betrachtet werden (Coltrane 2000; IshiiKuntz/Coltrane 1992) u.a. auch deshalb, weil die Betreuung der Kinder sowohl von Männern als auch von Frauen in der Regel positiver bewertet werden als Hausarbeitstätigkeiten (Gager 1998; Kroska 2003).

Geschlechtsunterschiede

Auch in unserer Studie leisteten die Frauen einen deutlich größeren Beitrag zur Hausarbeit und Kinderbetreuung als die Männer, während die Männer umgekehrt einen größeren Anteil an der Erwerbstätigkeit hatten. Bemerkenswert ist, dass nicht nur die Frauen sondern auch die Männer umso zufriedener mit der Aufteilung der Hausarbeit und Kinderbetreuung waren, je größer ihr eigener Anteil an der entsprechenden Tätigkeit war. Dieses Ergebnis, das sich am deutlichsten in den Korrelationen zeigt, bedeutet auch, dass Männer die weniger engagiert in der Hausarbeit und bei der Kinderbetreuung sind, diesen Zustand auch weniger befriedigend empfinden, möglicherweise auch deshalb, weil sie ein eher egalitäres Ideal der Arbeitsteilung befürworten.

Wie bereits erwähnt, gab es bezüglich der Hausarbeit signifikante Unterschiede zwischen Frauen und Männern. So zeigt sich im berechneten Modell, dass die Zufriedenheit der Frauen mit der Aufteilung der Hausarbeit deutlich höher war, wenn die Männer relativ gesehen einen größeren Anteil an der Hausarbeit übernahmen. Bei den Männern gab es diesen Zusammenhang dagegen nicht. Interessant ist auch, dass bei den Frauen die Zufriedenheit mit der Aufteilung der Hausarbeit höher war, wenn die Männer mehr Zeit für den Beruf aufwandten. Das heißt, dass die Frauen bei der Zufriedenheitsbeurteilung der Arbeitsteilung nicht nur den Einsatz ihrer Männer im Haushalt bewerten, sondern auch die beruflichen 
Verpflichtungen mitberücksichtigen. Bei den Männern spielen die Aufteilung der Hausarbeit und der Berufsarbeit für die Zufriedenheitseinschätzung dagegen keine Rolle.

Zudem war die Zufriedenheit mit der Aufteilung der Hausarbeit bei den Frauen signifikant höher als bei den Männern mit den wahrgenommenen Partnerschaftskonflikten assoziiert (Bewährung H-1.1 für die Hausarbeit) und hatte auch einen beträchtlichen direkten Effekt auf die Partnerschaftszufriedenheit, der bei den Männern nicht vorhanden war. Das heißt: mit der Aufteilung der Hausarbeit unzufriedene Frauen berichteten mehr Partnerschaftskonflikte, waren aber auch unabhängig davon unzufriedener mit ihrer Paarbeziehung. Ähnliche Ergebnisse wurden auch in anderen Untersuchungen gefunden (Essex/ Hong 2005; Kluwer et al. 1996; Stevens et al. 2001).

Bemerkenswert sind die Partnereffekte von der Zufriedenheit mit der Aufteilung der Hausarbeit auf das Konflikterleben des Partners: Männer berichteten mehr Konflikte, wenn ihre Frauen unzufrieden mit der Aufteilung der Hausarbeit waren. Dies trifft auch umgekehrt zu und macht deutlich, dass die Bewertung der Aufteilung direkte Effekte auf der Paarebene hat (Bewährung von H-1.2 für die Hausarbeit).

Einschränkungen der vorliegenden Studie

Neben dem Vorzug, dass die Daten von beiden Partnern erhoben und in einem differenzierten Aktor-Partner-Interdependenz-Modell statistisch geprüft wurden, weist die vorliegenden Untersuchung auch spezifische methodische Probleme auf, die nachfolgend diskutiert werden.

Bei den erhobenen Zeitangaben für die Familien- und Berufsarbeit ebenso wie bei der Bewertung der Zufriedenheit mit der Aufteilung, der Einschätzung der Konflikthäufigkeit und der Bewertung der Partnerschaftszufriedenheit handelt es sich um globale Einschätzungen. Diese können durch Erinnerungseffekte verzerrt sein (Fahrenberg et al. 2007; Schulz/Grunow 2007). Das bedeutet, dass wir in der vorliegenden Untersuchung die mentalen Repräsentationen (Perrez 2006) der Teilnehmer erfasst haben, d.h. beispielsweise die Vorstellung darüber, wie viele Stunden sie selbst oder ihr Partner während der letzten Woche investiert hat. Diese mentalen Repräsentationen können von der tatsächlichen Gegebenheit abweichen. Sie sind aber dennoch bedeutsam, da sie die Bewertung der entsprechenden Phänomene und letztlich auch das Verhalten der Partner beeinflussen.

Die in unserem Modell (Abbildung 1) spezifizierten Effekte sind gerichtet. Wir postulieren z.B., dass Paarkonflikte sich auf die Partnerschaftszufriedenheit auswirken. Allerdings wäre auch eine andere Richtung der Effekte denkbar: z.B. könnte eine geringere Partnerschaftszufriedenheit auch zu vermehrten Partnerschaftskonflikten führen. Da wir in unserer Studie lediglich zu einem Zeitpunkt Informationen erhoben haben, ist es nicht möglich, empirisch begründete Schlussfolgerungen über die kausale Richtung der Effekte zu ziehen.

Eine weitere Einschränkung ergibt sich aus der Zusammensetzung unserer Stichprobe. Untersucht wurden berufstätige Deutschschweizer Eltern mit Kleinkindern. Die Paare wiesen vorwiegend eine überdurchschnittliche Bildung und einen gehobenen sozioökonomischen Status auf. Im Hinblick auf die Zeit, die unsere Untersuchungsteilnehmer für Hausarbeit und Kinderbetreuung aufwandten, sind sie jedoch vergleichbar mit durchschnittlichen Schweizer Eltern, welche Kinder bis zu 6 Jahren haben und beide berufstätig sind (Bundesamt für Statistik 2009). 
Da sich die Schweiz, Österreich und Deutschland bezüglich der Entwicklung der Erwerbsquoten von Frauen sowie der Aufteilung der Erwerbs- und Familienarbeit kaum voneinander unterscheiden (Hofäcker 2007), gehen wir davon aus, dass die zentralen Ergebnisse der vorliegenden Untersuchung zumindest auf Doppelverdiener-Paare mit Kleinkindern in der Schweiz, Deutschland und Österreich zutreffen. Offen bleibt jedoch die Frage, ob sich die gefundenen Ergebnisse auch auf andere Paarformen (u.a. Doppelverdiener-Paare ohne Kinder oder Paare, bei denen nur die Männer berufstätig sind) übertragen lassen.

Trotz der genannten Einschränkungen kann als Fazit festgehalten werden, dass bei Doppelverdiener-Paaren weniger die tatsächliche Aufteilung der Hausarbeit und Kinderbetreuung für eine zufriedene Partnerschaft relevant ist, sondern mehr deren Bewertung. Die Zufriedenheit mit der familiären Arbeitsteilung wirkt sich sowohl direkt als auch vermittelt über das Konflikterleben auf die Partnerschaftszufriedenheit aus. Diese Effekte sind größer bei Frauen als bei Männern, wenn die Aufteilung der Hausarbeit beurteilt wird, nicht aber wenn die Aufteilung der Kinderbetreuung beurteilt wird.

\section{Literatur}

Bartley, S. J., Blanton, P. W. \& Gilliard, J. L. (2005). Husbands and wives in dual-earner marriages: Decision-making, gender role attitudes, division of household labor, and equity. Marriage and Family Review, 37, 4, S. 69-94.

Blair, S. L. (1998). Work roles, domestic roles, and marital quality: Perceptions of fairness among dualearner couples. Social Justice Research, 11, 3, S. 313-335.

Bonney, J. F., Kelley, M. L. \& Levant, R. F. (1999), A model of fathers' behavioral involvement in child care in dual-earner families. Journal of Family Psychology, 13, 3, S. 401-415.

Bundesamt für Statistik, Schweiz (2009). Familien Haushalte - Analyse: Statistischer Bericht 2008. http://www.bfs.admin.ch/bfs/portal/de/index/themen/01/04/blank/01/04/02.html, <12.10.2009>.

Chan, R. W., Brooks, R. C., Raboy, B. \& Patterson, C. J. (1998). Division of labor among lesbian and heterosexual parents: Associations with children's adjustment. Journal of Family Psychology, 12, 3, S. $402-419$.

Coltrane, S. (2000). Research on household labor: Modeling and measuring the social embeddedness of routine family work. Journal of Marriage and the Family, 62, 4, S. 1208-1233.

Ehrenberg, M. F., Gearing-Small, M., Hunter, M. A. \& Small, B. J. (2001). Childcare task division and shared parenting attitudes in dual-earner families with young children. Family Relations, 50, 2, S: 143-153.

Erickson, R. J. (1993). Reconceptualizing family work: The effect of emotion work on perceptions of marital quality. Journal of Marriage and the Family, 55, 4, S. 888-900.

Essex, E. L. \& Hong, J. (2005). Older caregiving parents: Division of household labor, marital satisfaction, and caregiver burden. Family Relations, 54, 3, S. 448-460.

Fahrenberg, J., Myrtek, M., Pawlik, K. \& Perrez, M. (2007). Ambulantes Assessment - Verhalten im Alltagskontext erfassen. Eine verhaltenswissenschaftliche Herausforderung an die Psychologie. Psychologische Rundschau, 58, S. 12-23.

Frisco, M. L. \& Williams, K. (2003). Perceived housework equity, marital happiness, and divorce in dual-earner households. Journal of Family Issues, 24, 1, S. 51-73.

Gager, C. T. (1998). The role of valued outcomes, justifications, and comparison referents in perceptions of fairness among dual-earner couples. Journal of Family Issues, 19, 5, S. 622-648.

Grote, N. K., Naylor, K. E. \& Clark, M. S. (2002). Perceiving the division of family work to be unfair: Do social comparisons, enjoyment, and competence matter? Journal of Family Psychology, 16, 4, S. 510-522. 
Grunow, D. (2007). Wandel der Geschlechtsrollenideologie und Väterhandeln im Alltag. In: T. Mühling \& H. Rost (Hrsg.), Väter im Blickpunkt. Perspektiven der Familienforschung. Opladen \& Farmington Hills, MI: Verlag Barbara Budrich, S. 49-76.

Hendrick, S. S. (1988). A generic measure of relationship satisfaction. Journal of Marriage and the Family, 50, 1, S. 93-98.

Himsel, A. J. \& Goldberg, W. A. (2003). Social comparisons and satisfaction with the division of housework: Implications for men's and women's role strain. Journal of Family Issues, 24, 7, S. 843-866.

Hochschild, A. R. (1989). The second shift. Working parents and the revolution at home. New York: Penguin Books.

Hoelter, L. F. (2002). Fair is fair - or is it? Perceptions of fairness in the household division of labor. Dissertation Abstracts International Section A: Humanities and Social Sciences, 62, 7, S. 2587.

Hofäcker, D. (2007). Väter im internationalen Vergleich. In: T. Mühling \& H. Rost (Hrsg.), Väter im Blickpunkt. Perspektiven der Familienforschung. Opladen \& Farmington Hills, MI: Verlag Barbara Budrich, S. 161-204.

Huinink, J. \& Reichart, E. (2008). Der Weg in die traditionelle Arbeitsteilung - eine Einbahnstraße? In: W. Bien (Hrsg.), Familiale Beziehungen, Familienalltag und soziale Netzwerke: Ergebnisse der drei Wellen des Familiensurvey. Wiesbaden: VS Verlag für Sozialwissenschaften, S. 43-79.

Ishii-Kuntz, M. \& Coltrane, S. (1992). Predicting the sharing of household labor: Are parenting and housework distinct? Sociological Perspectives, 35, 4, S. 629-647.

Kenny, D. A., Kashy, D. A. \& Cook, W. L. (2006). Dyadic data analysis. New York: The Guilford Press.

Kirchler, E. \& Venus, M. (2000). Zwischen Beruf und Familie: Gerechtigkeit und Zufriedenheit mit der Aufteilung der Arbeit zu Hause. Zeitschrift für Sozialpsychologie, 31, 2, S. 113-123.

Klaus, D. \& Steinbach, A. (2002). Determinanten innerfamiliärer Arbeitsteilung. Eine Betrachtung im Längsschnitt. Zeitschrift für Familienforschung, 14, 1, S. 21-43.

Kluwer, E. S., Heesink, J. A. M. \& Van de Vliert, E. (1996). Marital conflict about the division of household labor and paid work. Journal of Marriage and the Family, 58, 4, S. 958-969.

Kroska, A. (2003). Investigating gender differences in the meaning of household chores and child care. Journal of Marriage and the Family, 65, S. 456-473.

Koppetsch, C. \& Burkart, G. (1999). Die Illusion der Emanzipation. Zur Wirksamkeit latenter Geschlechtsnormen im Milieuvergleich. Konstanz: Universitätsverlag.

Ledermann, T. \& Bodenmann, G. (2006). Moderator- und Mediatoreffekte bei dyadischen Daten. Zwei Erweiterungen des Akteur-Partner-Interdependenz-Modells. Zeitschrift für Sozialpsychologie, 37, 1, S. 27-40.

Levy, R. \& Ernst, M. (2002). Lebenslauf und Regulation in Paarbeziehungen: Bestimmungsgründe der Ungleichheit familialer Arbeitsteilung. Zeitschrift für Familien-forschung, 14, 2, S. 103-131.

Lothaller, H., Mikula, G. \& Schoebi, D. (2009). What contributes to the (im)balanced division of family work between the sexes? Swiss Journal of Psychology, 68, 3, S. 143-152.

Meuwly, N. (2007). Zufriedenheit mit der Arbeitsteilung der Hausarbeit und Kinderbetreuung, und ihre Auswirkungen auf die Partnerschaftszufriedenheit. Eine Untersuchung bei Doppelverdiener-Paaren. Fribourg: Universität Fribourg (unveröffentlichte Lizentiatsarbeit).

Mikula, G., Riederer, B. \& Bodi, O. (2008). Women's professional and familial work loads, relationship satisfaction and well-being: The mediating role of subjective work loads and perceived justice of the division of family work. In: A.M. Fontaine \& M. Marias (Hrsg.), Work, family and personal dynamics: International Perspectives. Porto: Legis/LivPsic, S. 65-78.

Muthén, L.K. \& Muthén, B. O. (1998-2007). Mplus user's guide. Los Angeles, CA: Muthén \& Muthén ( $5^{\text {th }}$ edition).

Perrez, M. (2006). Plädoyer für theorieadäquate Methoden in gewissen Domänen der Psychologie. Verhaltenstherapie und Psychosoziale Praxis, 38, 2, S. 319-330.

Rohmann, E., Schmohr, M. \& Bierhoff, H.-W. (2002). Aufteilung der Hausarbeit, verletzte Erwartungen und Beziehungsqualität. Zeitschrift für Familienforschung, 14, 2, S. 133-152. 
Sander, J. \& Böcker, S. (1993). Die deutsche Form der Relationship Assessnent Scale (RAS): eine kurze Skala zur Messung der Zufriedenheit in einer Partnerschaft. Diagnostica, 39, S. 55-62.

Schulz, F. \& Blossfeld, H.-P. (2006). Wie verändert sich die häusliche Arbeitsteilung im Eheverlauf? Kölner Zeitschrift für Soziologie und Sozialpsychologie, 58, 1, S. 23-49.

Schulz, F. \& Grunow, D. (2007). Tagebuch versus Zeiteinschätzung. Ein Vergleich zweier unterschiedlicher Methoden zur Messung der Zeitverwendung für Hausarbeit. Zeitschrift für Familienforschung, 19,1, S. 106-128.

Shrout, P. E. \& Bolger, N. (2002). Mediation in experimental and nonexperimental studies: New procedures and recommendations. Psychological Methods, 7, 4, S. 422-445.

Stevens, D. P., Kiger, G. \& Mannon, S. E. (2005). Domestic labor and marital satisfaction: How much or how satisfied? Marriage and Family Review, 37, 4, S. 49- 67.

Stevens, D., Kiger, G. \& Riley, P. J. (2001). Working hard and hardly working: Domestic labor and marital satisfaction among dual-earner couples. Journal of Marriage and Family, 63, 2, S. 514-526.

Voydanoff, P. \& Donnelly, B. W. (1999). The intersection of time in activities and perceived unfairness in relation to psychological distress and marital quality. Journal of Marriage and the Family, 61, 3, S. 739-751.

Wilkie, J. R., Feree, M. M. \& Ratcliff, K. S. (1998). Gender and fairness: Marital satisfaction in twoearner couples. Journal of Marriage and the Family, 60, 3, S. 577-594.

Yogev, S. \& Brett, J. (1985). Perceptions of the division of housework and child care and marital satisfaction. Journal of Marriage and the Family, 47, 3, S. 609-618.

Eingereicht am/Submitted on: 06.04.2009

Angenommen am/Accepted on: 18.05.2010

Anschrift der Autor(inn)en/Address of the authors:

lic. phil. Nathalie Meuwly, Universität Zürich

Dr. phil. Peter Wilhelm, Universität Fribourg

lic. phil. Véronique Eicher, Universität Fribourg und Universitäre Fernstudien Schweiz

Prof. Dr. Meinrad Perrez, Universität Fribourg und Universitäre Fernstudien Schweiz

lic. phil. Nathalie Meuwly (Korrespondenzadresse/corresponding author)

Universität Zürich

Psychologisches Institut

Lehrstuhl für Klinische Psychologie

mit Schwerpunkt Kinder/Jugendliche und Paare/Familien

Binzmühlestrasse 14/23

8050 Zürich

Schweiz/Switzerland

Email: nathalie.meuwly@psychologie.uzh.ch 